\title{
Dietary krill oil significantly reduces hepatic steatosis, glycaemia and hypercholesterolaemia in high-fat-fed mice
}

\author{
S. Tandy ${ }^{1}$, R. W.S. Chung ${ }^{1}$, E. Wat ${ }^{1}$, A. Kamili ${ }^{1}$, M. Griinari ${ }^{2}$, K. Berge ${ }^{2}$ and J.S. Cohn ${ }^{1}$ \\ ${ }^{1}$ Heart Research Institute, Sydney, NSW, Australia and ${ }^{2}$ Aker BioMarine ASA, Oslo, Norway
}

Dietary krill oil (KO), rich in $n$-3 fatty acids associated with phospholipid, is extracted from Antarctic krill (Euphausia superba). Taken as a dietary supplement, it is believed to have important anti-inflammatory and cardioprotective properties. ${ }^{(1,2)}$. In order to investigate the effects of $\mathrm{KO}$ on lipid and glucose metabolism, the present study was carried out in mice fed a high-fat diet supplemented with different doses of $\mathrm{KO}$.

Male C57BL/6 mice were fed a high-fat (HF) diet (w/w; $21 \%$ butterfat, $0.15 \%$ cholesterol) supplemented with $0,1.25,2.5$ or $5 \%$ (w/w) dietary KO. Animals (six to ten per group) were killed after 8 weeks. Livers were analysed for lipid content and serum samples for lipids, glucose and adiponectin.

Dietary KO supplementation caused a significant reduction in liver weight (i.e. hepatomegaly) and total liver fat (i.e. hepatic steatosis) as a result of a dose-dependent reduction in hepatic TAG ( 35 (SE 6) \%, 47 (SE 4) \% and 51 (SE 5) \% with KO supplementation of $1.25,2.5$ and $5.0 \%$ respectively; $P<0.001)$ and cholesterol (55 (SE 5) \%, 66 (SE 3) \% and 71 (SE 3) \% with KO supplementation of $1.25,2.5$ and $5.0 \%$ respectively; $P<0.001)$.

\begin{tabular}{|c|c|c|c|c|c|c|c|c|c|c|}
\hline & & & \multicolumn{8}{|c|}{$\mathrm{HF}(\% ; \mathrm{w} / \mathrm{w})$} \\
\hline & \multicolumn{2}{|c|}{$\mathrm{N}$} & \multicolumn{2}{|c|}{0} & \multicolumn{2}{|c|}{1.25} & \multicolumn{2}{|c|}{2.5} & \multicolumn{2}{|c|}{5.0} \\
\hline & Mean & $\mathrm{SE}$ & Mean & $\mathrm{SE}$ & Mean & $\mathrm{SE}$ & Mean & $\mathrm{SE}$ & Mean & $\mathrm{SE}$ \\
\hline Total fat: $\mathrm{g} / 100 \mathrm{~g}$ & 7 & 1 & $18^{* *}$ & 4 & $11 \dagger$ & 1 & $9 \dagger \dagger$ & 1 & $9+\dagger$ & 1 \\
\hline mg/organ & 65 & 5 & $290 * *$ & 86 & $140 \dagger$ & 17 & $116+\dagger$ & 9 & $116+\dagger$ & 13 \\
\hline TAG: $\mu \mathrm{mol} / \mathrm{g}$ & 39 & 4 & $146^{* * *}$ & 31 & 95 & 10 & $77+\dagger$ & 5 & $72+\dagger$ & 7 \\
\hline$\mu \mathrm{mol} /$ organ & 39 & 4 & $234 * * *$ & 61 & $121 \dagger$ & 15 & $98+\dagger$ & 8 & $93+\dagger$ & 13 \\
\hline Cholesterol: $\mu \mathrm{mol} / \mathrm{g}$ & 7 & 1 & $38 * *$ & 10 & $17 \dagger \dagger$ & 2 & $13+\dagger \dagger$ & 1 & $11+\dagger \dagger$ & 1 \\
\hline$\mu \mathrm{mol} /$ organ & 7 & 1 & $62 * *$ & 20 & $22 \dagger \dagger$ & 3 & $16 \dagger \dagger$ & 1 & $14+\dagger \dagger$ & 2 \\
\hline Phospholipid: $\mu \mathrm{mol} / \mathrm{g}$ & 13 & 1 & 14 & 1 & 12 & 1 & $11 \dagger$ & 1 & 12 & 1 \\
\hline$\mu \mathrm{mol} /$ organ & 13 & 1 & $21^{* *}$ & 3 & $15 \dagger$ & 1 & $14 \dagger \dagger$ & 1 & $15 \dagger$ & 1 \\
\hline
\end{tabular}

$\mathrm{N}$, normal-fat diet. Mean values were significantly different from those for the HF diets (Student's $t$ test: $* * P<0.01, * * * P<0.001$. Mean values were significantly different from those for HF-KO-supplemented groups (ANOVA followed by Tukey's test for significance): $\dagger P<0.05, \dagger \dagger P<0.01, \dagger \dagger \dagger P<0.001$.

Serum cholesterol levels were reduced by 20 (SE 3) \%, 29 (SE 4) \% and 29 (SE 5) \% and blood glucose was reduced by 36 (SE 5) \%, 34 (SE 6) \% and 42 (SE 6) \% with KO supplementation at 1.25, 2.5 and 5.0\% respectively. Serum adiponectin was increased in KO-fed animals (HF $v$. HF-KO at $5.0 \%, 4.97$ (SE 0.18) $\mu \mathrm{g} / \mathrm{ml} v .7 .43$ (SE 0.61$) \mu \mathrm{g} / \mathrm{ml} ; P<0.01$ ).

The data demonstrate that in high-fat-fed mice dietary KO reduces serum cholesterol and glucose levels, as well as having a beneficial effect on hepatic lipid metabolism. These results support the concept that dietary KO may be of therapeutic benefit in individuals at increased risk of the metabolic syndrome and associated non-alcoholic fatty liver disease.

1. Deutsch L. (2007) Evaluation of the effect of Neptune Krill Oil on chronic inflammation and arthritic symptoms. J Am Coll Nutr 26, 39-48.

2. Bunea R, El Farrah K \& Deutsch L. (2004) Evaluation of the effects of Neptune Krill Oil on the clinical course of hyperlipidemia. Altern Med Rev 9, 420-428. 